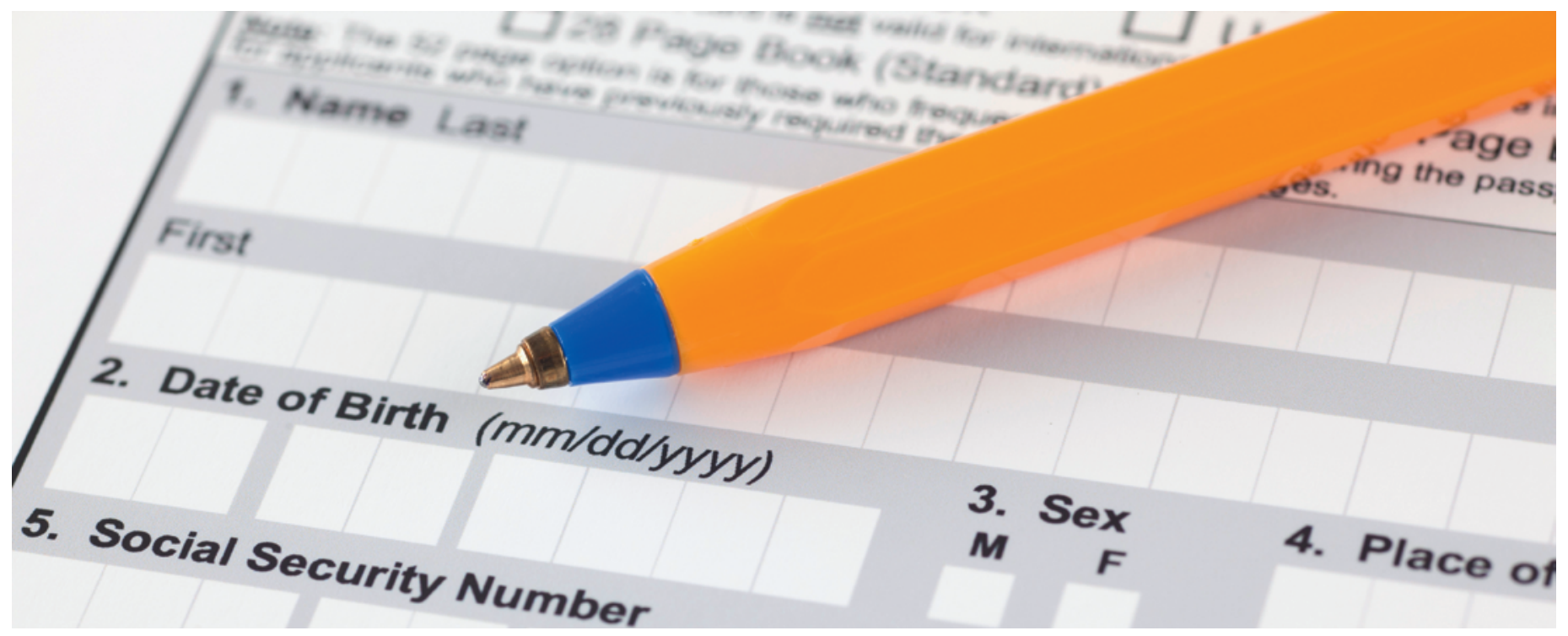

\title{
Détermination de l'âge des jeunes migrants
}

\section{Sarah Depallens ${ }^{a}$, Fabienne Jäger ${ }^{b}$, Nicole Pellaud ${ }^{c}$}

${ }^{a}$ Dr méd., Service de pédiatrie, CHUV, Lausanne, et groupe migrants SSP, membre FMH; b Dr méd., Service de pédiatrie, Hôpital du Jura, Delémont, et groupe migrants SSP, membre FMH; ${ }^{c}$ Dr méd., Présidente SSP, Service médical scolaire et psychopédagogique, Sion, membre FMH

Cette position a été validée par les experts de pédiatrie du développement, Prof. O. Jenni, d'endocrinologie pédiatrique, Prof. V. Schwitzgebel, de radiologie pédiatrique, G. Eich, ainsi que par le comité de la Société suisse de pédiatrie et son groupe santé migrants, www.swiss-paedaitrics.org

\footnotetext{
1 Pour faciliter la lecture, la forme masculine est utilisée pour représenter les deux genres.
}

La Société suisse de pédiatrie (SSP) souhaite informer les médecins ainsi que les instances fédérales et cantonales compétentes de la problématique qui se pose à propos de la détermination de l'âge des jeunes migrants ${ }^{1}$. Elle publie une position élaborée par des pédiatres spécialisées en santé des jeunes migrants et en pédiatrie sociale. En souhaitant que cette position puisse promouvoir en Suisse une prise en charge des jeunes migrants respectueuse des droits de l'enfant et des droits de l'homme.

\section{Sans méthode validée, les médecins ne peuvent pas y participer}

Chaque année, la Suisse accueille des requérants d'asile. Ils étaient 27207 en 2016 dont 7,3\% considérés comme des mineurs non accompagnés [1].

Parallèlement à d'autres critères, la procédure d'asile tient compte de l'âge du jeune requérant, les adolescents de plus de 18 ans n'ayant pas droit à l'encadrement réservé d'office aux mineurs (foyer avec éducateurs, protection par un tuteur, accès à l'école,...). Cette différence de traitement a un impact important pour le futur du jeune. Par exemple, l'accès à une formation étant un des facteurs protecteurs les plus importants à l'adolescence, un mineur estimé comme majeur à son arrivée en Suisse sera dès son départ à risque de désinsertion et donc en danger dans son développement. En Suisse, afin de déterminer l'âge des jeunes qui ne possèdent pas de documents valables attestant leur date de naissance, les institutions gouvernementales font parfois appel aux médecins.

Sur le plan de la déontologie médicale, tout examen n'est réalisé qu'au respect des conditions suivantes: 1) indication médicale pour l'amélioration de la santé, 2) consentement éclairé de la personne, 3) méthode validée et sans danger [2,3]. 
Dans le cadre d'un mandat non thérapeutique, la personne doit être clairement informée et le médecin ne peut pas avoir recours à des pratiques diagnostiques discutables [3].

En Suisse, afin de déterminer l'âge des jeunes qui ne possèdent pas de documents valables attestant leur date de naissance, les institutions gouvernementales font parfois appel aux médecins.

Or, pour ces déterminations, comme cela a déjà été démontré et publié à diverses reprises [4, 5], que cela soit pour l'âge osseux $[6-8,11]$, l'examen physique [8] ou dentaire $[9,10,12]$, ces outils sont trop approximatifs et présentent des écarts-types larges, ils se fondent sur des tables de références souvent non adaptées car ne tenant compte ni de l'origine ethnique $[11,12]$ ou socioéconomique du jeune, ni d'éventuelles pathologies endocriniennes [8] pouvant influencer les résultats. S’y ajoute une irradiation inutile.

Aujourd'hui, aucune méthode scientifique ne permet d'établir précisément l'âge d'un jeune qui se situerait entre 15 et 20 ans afin de définir avec certitude s'il est majeur ou mineur: en effet des valeurs d'adultes peuvent être trouvées chez un jeune mineur conduisant à une surévaluation de son âge.

Une évaluation globale par des professionnels spécialisés dans le développement de l'adolescent reste pertinente afin d'évaluer le développement du jeune, son statut psycho-cognitif, et de pouvoir si nécessaire

\section{Aujourd'hui, aucune méthode scientifique ne permet d'établir précisément l'âge d'un jeune qui se situerait entre 15 et 20 ans.}

l'orienter vers des structures qui puissent tenir compte de ses vulnérabilités [13].

Dans un contexte où la détermination de l'âge ne répond pas aux critères déontologiques et comporte un risque d'erreur avec des conséquences légales et sociales majeures sur la vie de ces jeunes, la Société suisse de pédiatrie, comme l'ont déjà fait plusieurs sociétés et académies internationales de pédiatrie [14-16], recommande à ses membres et à tout médecin sollicité de ne pas participer à la détermination de l'âge des jeunes requérants d'asile et de prendre position dans ce sens auprès des institutions cantonales en charge de la migration.

\section{Références}

1 https://asile.ch/statistiques/suisse/

2 Charte du pédiatre, SSP 2017.

3 Code de déontologie FMH art. 6 et 8, http://www.fmh.ch/files/ pdf18/Standesordnung_August_2016_F.pdf

4 Aynsley-Green A, Cole TJ, Crawley H, Lessof N, Boag LR, Wallace RM. Medical, statistical, ethical and human rights considerations in the assessment of age in children and young people subject to immigration control. British Medical Bulletin. Jun 2012;102:17- 42 .

5 Hjern A, Brendler-Lindqvist M, Norredam M. Age assessment of young asylum seekers. Acta paediatrica (Oslo, Norway: 1992). Jan 2012;101(1):4-7.

6 Cole TJ. The evidential value of developmental age imaging for assessing age of majority. Annals of human biology. 2015;42(4):379-88.

7 Thodberg Hans Henrik, van Rijn Rick R, Jenni Oskar G, Martin David. D. Automated determination of bone age from hand Xrays at the end of puberty and its applicability for age estimation Int J Legal Med. DOI 10.1007/s00414-016-1471-8

8 Schwitzgebel V. Georg F. L'âge osseux ne permet pas de déterminer l'âge des jeunes requérants d'asile, Paediatrica Vol. 27 No. 3, 292016

9 Knell B. 2012. Zahnärztliche Altersdiagnostik zur Frage nach dem 18. Altersjahr. Kriminalistik-Schweiz. 2/12, 122-127, 2012

10 Reutimann, Felix. Zahnärztliche Altersdiagnostik: Untersuchung zur radiologischen Sichtbarkeit des Parodontalspaltes der ersten Molaren im Unterkiefer bei 14- bis 22-Jährigen. 2015, University of Zurich, Faculty of Medicine. http://www.zora.uzh. ch/123990/1/Dissertation_Reutimann_12_12_2015.pdf

11 Ontell FK, Ivanovic M, Ablin DS and Barlow TW. Bone age in children of diverse ethnicity. http://www.ajronline.org/doi/ abs/10.2214/ajr.167.6.8956565

12 Olze A, Schmeling A, Taniguchi M, Maeda H, van Niekerk P Wernecke KD, Geserick G. Forensic age estimation in living subjects: the ethnic factor in wisdom tooth mineralization, Int J Legal Med. 2004 Jun;118(3):170-3. Epub 2004 Feb 6.

13 Messelken D, Crouse J. When childhood ends: estimating the age of young people. BMJ. 2015;351:h6699 doi: 10.1136/bmj.h6699

14 Royal College of Paediatrics and Child Health (2009) Policy: College statement on the role of paediatricians in the age assessment of unaccompanied young people seeking asylum. http:// www. rcpch.ac.uk/Policy

15 On behalf of the Advocacy and Ethics Group of the European Academy of Paediatrics, Sauer PJJ, Nicholson A, Neubauer D. Age determination in asylum seekers: physicians should not be implicated. Eur J Pediatr. Mar 2016; 175(3), 299-303 (2016).

16 International Society for Social Paediatrics Position Statement on Migrant Child Health 2017.

\section{Crédit photo}

(c) Ekaterina79 | Dreamstime.com 